\title{
Determination of Water Content in Ethanol by Miniaturized Near-Infrared (NIR) System
}

\author{
Soohwa Cho, Hoeil Chung, ${ }^{*}$ Young-Ah Woo, ${ }^{\dagger}$ and Hyo-Jin Kim ${ }^{*}$ \\ Deparment of Chemistr; College of Natural Sciences, Hanvang Cniversity. Seoul 133-791. Korea \\ E-mail: hoeilohamvang ackr \\ 'College of Phamacy, Dongduk Women's Chiversirv. Seoul $136-814$, Korea \\ Received October 13, 2004
}

\begin{abstract}
The miniaturized NIR (Near-infrared) spectrometer has been utilized for the determination of water content (1$19 \%$ range) in ethanol that is the most popular organic solvent in pharnaceutical industries. It has many potential capabilities that can replace the conventional analyzers especially for the on-line measurement since it is compact. versatile and cost-effective. By using two dimensional (2D) correlation spectroscopy. it was preliminarily investigated to find any unforeseen spectral distortion among the spectra collected from the miniaturized spectrometer. The 2D study revealed that the spectral variation clearly followed the variation of water concentration without any spectral distortion or abnomality. PLS (Partial Least Squares) was employed to build the calibration model and the resulting prediction perfornance was acceptable and stable over several days. Even though the miniaturized NIR system was evaluated to fairly simple chenical matrix, the overall study demonstrates the sufficient feasibility for diverse practical and industrial applications.
\end{abstract}

Key Words : Near-infrared spectroscopy. Miniaturized spectrometer. Ethanol solution, 2D correlation spectroscopy

\section{Introduction}

Ethanol is one of the most popular organic materials in the pharmaceutical industry for use as a general solvent as well as an extraction agent. Due to its heavy usage. it is usually purified and regenerated by reçcling used ethanol. The majority of the used ethanol stream contains methanol and water as impurities. Therefore. for optimal recycling of ethanol. the continuous monitoring of the water content is very important. Karl Fisher titration has been employed at large for this purpose. However. this method is very slow. destructive and easily subject to human errors. Near-infrared (NIR) spectroscopy i.: is an excellent analytical tool used to replace the wet analy'sis method because it is fast nondestructive and capable of continuous on-line measurement. ${ }^{3.4}$ In addition. it has been applied for the analysis of various alcoholic beverages such as beer. wines and distilled liquors. ${ }^{1}$ Recently. the development of the small-scale NIR instrument has gained widespread attention since it is compact relatively cheap and versatile in use.

In this study. a miniaturized NIR spectrometer has been developed and investigated for the determination of water content in the methanol/ethanol matrix. This system is composed of a tungsten-halogen lamp. a transflectance-type fiber optic sample probe a non-moving self-focusing grating and a photo-diode array (PDA) detector. There are no moving components in this system. The detailed instrumental description can be found in the previous publication. ${ }^{5}$ NIR spectra were collected for the samples ( $1 \%$ to $19 \%$ water content) and quantitative analysis was performed using the partial least squares (PLS) regression method. ${ }^{6-10}$ Additionally two dimensional (2D) correlation spectros- copy $y^{11-13}$ was employed to confirm the systematic spectral variation based on the concentration dependence. Overall results demonstrate that our miniaturized NIR system has a strong potential for use in many industrial on-line applications.

\section{Experimental Section}

Sample preparation: High purity anhy'drous ethanol and methanol ( $99.9 \%$ purity) were used. Initially an ethanol/ methanol mixture (of constant concentration) was prepared along with samples of various water concentrations. Ten samples from $1 \%$ to $10 \%$ (in $1 \%$ increments) and five samples from $11 \%$ to $19 \%$ (in $2 \%$ increments) were prepared. In total. 15 samples were prepared and used as the calibration sets. For validation. $3 \%, 5 \%$ and $7 \%$ samples were newly prepared each day and predicted over 5 days. All the NIR spectra were collected at room temperature.

Miniaturized NIR system and data processing: The overall schematic diagram of the miniaturized spectrometer is shown in Figure 1 and the more in-depth instrumental description is available elsewhere. ${ }^{5}$ The major component was the micro-spectrograph including the self-focusing diffraction grating with fiber fixing grooves fabricated by the combined use of lithography and micro-molding technologies. The light launched from the optical fiber is illuminated to the surface of the grating. An InGaAs photodiode array (PDA) with a 450 sidewall at the focus line was used for the signal detection. The spectral resolution of approximately $7 \mathrm{~nm}$ is achieved with the grating constant of 2-5 $\mathrm{fm}$ and the step height of $0.2-0.6 \mathrm{\mu m}$. The size of spectrograph was $2.5 \mathrm{~cm} \times 1.5 \mathrm{~cm} \times 0.1 \mathrm{~cm}$. The overall 


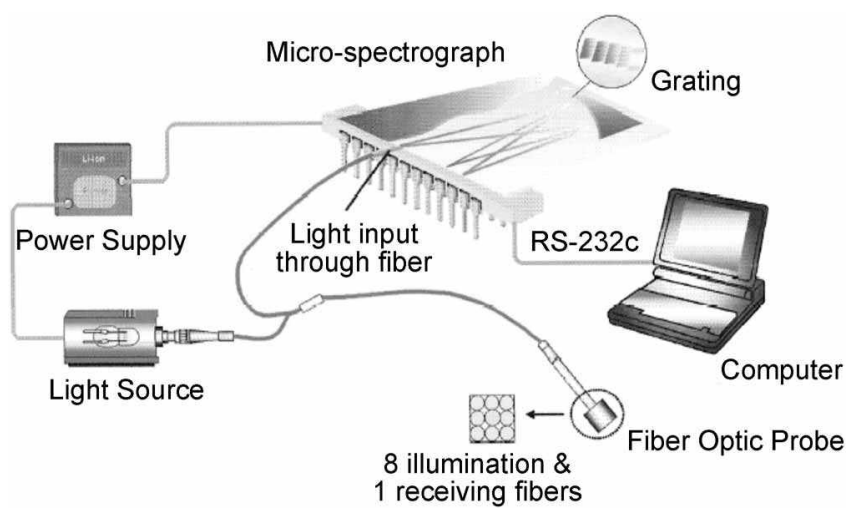

Figure 1. The schematic diagram of the miniaturized spectrometer.

system included a tungsten halogen lamp as a light source. a photodiode array for micro-spectrograph. and an extemal power supply (or internal battery). The necessary software was installed for data collection. The RS-232c cable was used to connect the NIR system with a computer.

The spectra were collected using a fiber optic transflectance probe. The probe consisted of eight illuminating fibers, a receiving fiber and a reflecting mirror. The distance between the optical fibers and the reflecting mirror was 1 mm. providing an actual patllength of a $2 \mathrm{~mm}$. Each spectrum corresponds to an accumulation of 30 scans in the $1100-1750 \mathrm{~nm}$ range with $2 \mathrm{~nm}$ data interval. Temperature of the detector was controlled at $30^{\circ} \mathrm{C}$.

A PLS regression and second derivative algorithm were performed using GRAMS/32 software with an add-on PLS algoritlum (Galactic Industries Corporation. Salem. NH).

\section{Results and Discussion}

Spectral features: Figure 2 (top) shows the NIR spectra of water mixtures taken by the miniaturized spectrometer. From the bottom spectra to the top spectra. the water concentration increased from $1 \%$ to $19 \%$. Water band $(\mathrm{OH})$ at $1450 \mathrm{~mm}\left(6900 \mathrm{~cm}^{-1}\right)$ was clearly observed to have increased with concentration. This band is the first overtone band of the $\mathrm{OH}$ stretching mode in the infrared region (e.g. $\approx 3450 \mathrm{~cm}^{-1} \times 2=6900 \mathrm{~cm}^{-1}=1450 \mathrm{~nm}$ ) that can be assigned to the hydrogen bonded $\mathrm{OH}$ peak. The band at $1580 \mathrm{~mm}$ is the free $\mathrm{OH}$ which is the first overtone peak from the methanol/ethanol mixture as well as water. The bands at 1180 and $1700 \mathrm{~nm}$ correspond to the second and first overtone of the $\mathrm{CH}$ stretching mode. As shown in this figure. the water band intensity at $1450 \mathrm{~mm}$ increased with the increase of water concentration.

To enhance the spectral features as well as to reduce the baseline variations. all the spectra were converted to their second derivative spectra. ${ }^{14}$ The corresponding second derivative spectra are shown in Figure 2 (bottom). The baseline variations were effectively removed and spectral features were enhanced while keeping the same qualitative information. The spectral variations based on water concentration were observed and the isosbestic point at $1476 \mathrm{~nm}$
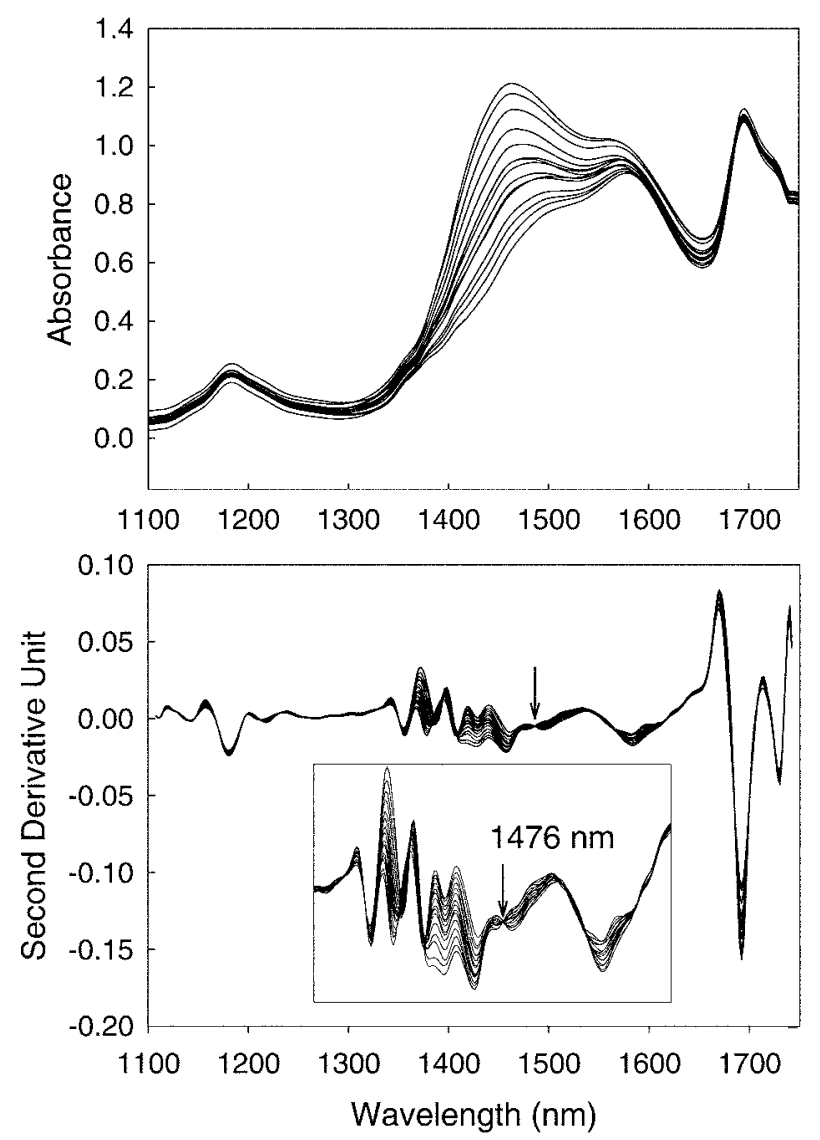

Figure 2. The raw NIR (top) and second derivative spectra (b) of $1 \%$ to $19 \%$ water content acquired from the miniaturized spectrometer.

between water and the methanol/ethanol mixture was identified (marked as arrow in the figure).

Two dimensional (2D) correlation spectroscopy: To investigate the performance of miniaturized spectrometer in more detail, two dimensional (2D) correlation spectroscopy $^{\text {ll-13 }}$ was employed. This method is very effective in finding the șistematic correlations in a given șistem under external perturbation. The external perturbation in this study was the variation of water concentration. Figure 3 shows the resulting contour map of synchronous $2 \mathrm{D}$ spectrum. The synchronous spectrum represents the concurrent change of spectral intensity at the 1100-1750 nm range. The spectral intensity variation on the diagonal line (centered at $1450 \mathrm{num}$. designated as A) is called "autopeak" that indicates the greater spectral change based on the concentration variation. The autopeak had consistent positive correlation (in this case an increase with concentration). Three cross peaks (off-diagonal positions) were also observed (B. C and D). A solid line $(B)$ and dotted lines $(C$ and $D)$ correspond to the positive and negative cross peaks. respectively. The peak $B$ had a positive correlation with the increase in water concentration. This peak position is near the free $\mathrm{OH}$ band from the methanol/ethanol mixture as well as water. ${ }^{15}$ It increased with the decrease of concentration of the ethanol/ methanol mixture. This indicates that the variation of water absorption is more dominant than those from the ethanol 


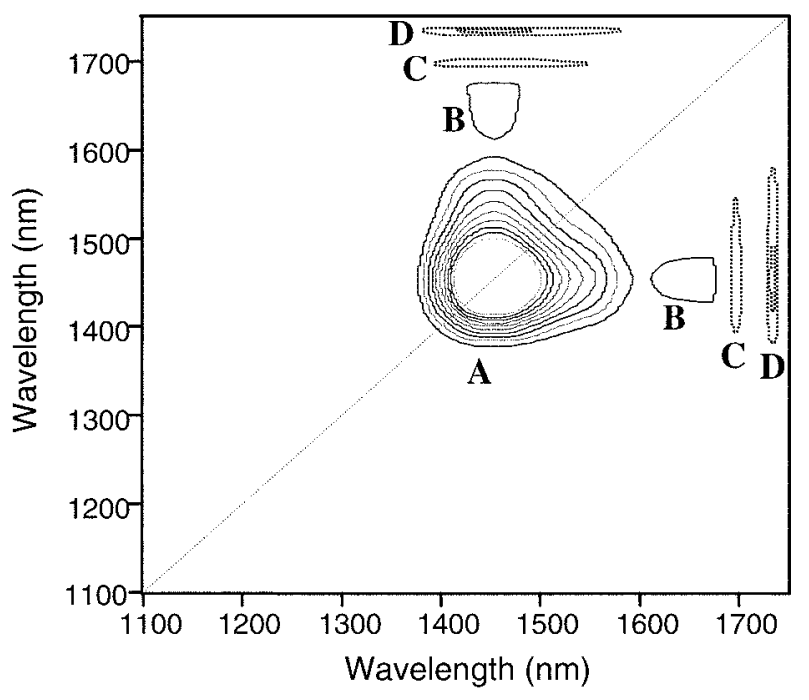

Figure 3. The contour map of synchronous two dimensional correlation spectrum.

methanol mixture due to the higher molar absorptivity of water. If the molar absorptivity of water is lower than those of ethanol/methanol it would show negative correlation from the decrease of ethanol/methanol concentration. Peaks C and D (hydrocarbon peaks of ethanol/methanol located around $1700 \mathrm{~nm}$ ) show the apparent negative correlation from the decrease of ethanol/methanol concentration and increase of water concentration. simultaneously. Overall the spectra collected from the miniaturized spectrometer depicted the expected spectral variation without a sign of spectral distortion. If spectral distortion were present. it could be easily detected by ambiguous correlation using 2D correlation spectroscopy:

PLS models: PLS regression ${ }^{(-1)}$ was used to build a calibration model. In the application of the PLS algorithm. it is generally known that the spectral range and the number of PLS factors are important parameters. ${ }^{16}$ In this research. the 1120-1730 $\mathrm{nm}$ range including the hydrocarbon and hydroxyl information was used. Then. PLS regression was performed using the second derivative spectra.

The optimum number of factors were identified as those that gave a minimum SECV (standard error of cross validation) and 3 PLS factors. in this case. The achieved SECV was $0.16 \%$. To validate the calibration model. three samples $(3 \% .5 \%$ and $7 \%$ ) were prepared new each day and predicted over 5 days (totaling 15 spectra: 3 samples $\times 5$ days). The concentration range from $3 \%$ to $7 \%$ was solely chosen for the prediction since the concentration variation is

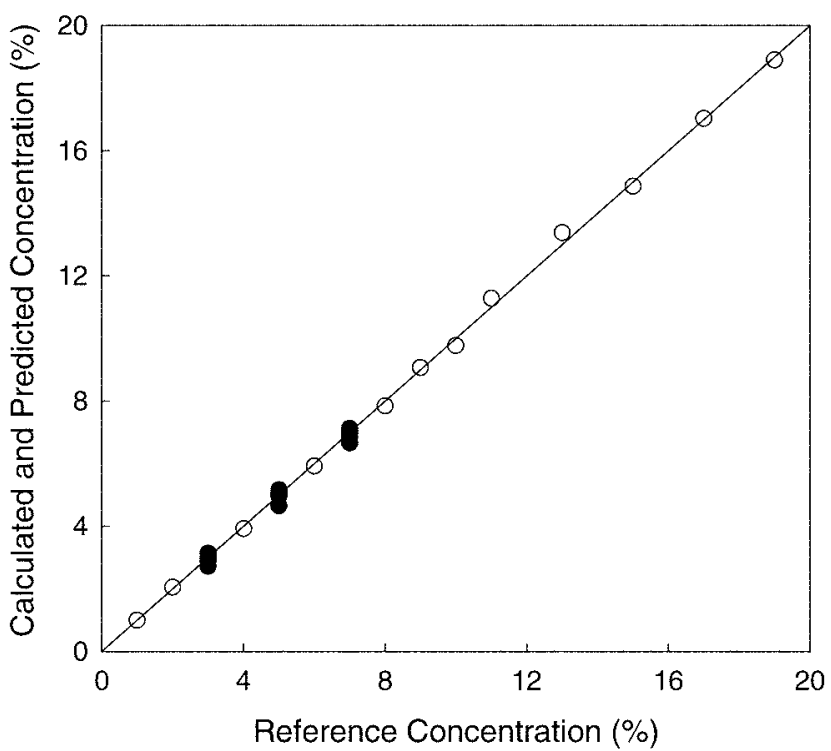

Figure 4. The scatter plot showing correlation between reference and NIR analyses. Open and filled circles represent the calibration and prediction data.

actually within this range. The prediction results are summarized in Table 1.

The prediction of each concentration over five days was nearly repeatable. with the standard deviation range from $0.15 \%$ to $0.19 \%$. As shown, there was no bias in the prediction with SEP (Standard Error of Prediction) of $0.16 \%$. The corresponding scatter plot showing correlation between reference and NIR analyses is shown in Figure 4. Open and filled circles represent the calibration and prediction data, respectively. The calibration and prediction data correlate very well with the reference data.

\section{Conclusions}

The overall results demonstrate the applicability of miniaturized NIR spectrometer in diverse fields. particularly in an on-line manner. The compact and cost-effective miniaturized NIR system could relieve the econonic burden for end-users. This study was based on a fairly simple chemical matrix. Future research will be directed to investigate its performance on highly complex chemical matrices such as refinery products (e.g. gasoline. diesel fuel. etc.).

Acknowledgements. This work is supported by the research fund from Research Institute for Natural Sciences at Hanyang University in 2002.

Table 1. PLS prediction results ( $3 \%, 5 \%, 7 \%$ water concentration) over 5 days

\begin{tabular}{cccccccc}
\hline $\begin{array}{c}\text { Reference } \\
\text { Concentration }\end{array}$ & Day 1 & Day 2 & Day 3 & Day 4 & Day 5 & Average & $\begin{array}{c}\text { Standard } \\
\text { Deviation }\end{array}$ \\
\hline 3.0 & 2.9 & 2.7 & 3.1 & 3.0 & 2.9 & 2.9 & 0.15 \\
5.0 & 5.2 & 4.7 & 5.0 & 5.0 & 5.0 & 5.0 & 0.19 \\
7.0 & 7.1 & 6.7 & 7.0 & 7.0 & 7.0 & 7.0 & 0.17 \\
\hline
\end{tabular}




\section{References}

1. Burns. D. A.: Ciurczak. E. W. Handbook of Near-Infrared Anatysis: Marcel Dekker Inc. New York. 1992.

2. Wetzel. D. L. Anal Chem. 1983. 55. 1165A.

3. Kamat, M. S.: Lodder, R. A.: DeLuca. P. P. Pham. Res. 1989. 6. 961.

4. Broad, N. W: Jee, R. D.: Moftat. A. C. Eaves. M. I.; Mann. W. C.: Dziki. W. Anallst 2000. 125.2054

5. Woo. Y. A.: Kim. H. J. Liseful and Adwanced Information in the field of Near Infrared Spectroscopv: Research Signpost: Kerela. India. 2003: chap. 14.

6. Mark H. Anal Chem 1986, 58,2814

7. Lee, S. H. Nam. J. J. Son, B. M. Bull. Korem them. Soc. 2003.
$24(2), 246$

8. Haaland. D. M.: Thomas. E. V. Anal. Chem. 1988. 60. 1202.

9. Martens. H.: Naes. T. M. Multivariate Calibration: Iohn Wiley and Son1s: New York. 1989

10. Beebe. K. R.: Pell, R. J.; Seasholtz. M. B. Chemometrics: A Practical Guide: John Wiley and Sons: New York. 1998.

11. Noda. I. Appl. Spectrosc. 1993, 47, 1329.

12. Noda. I.: Dowrey. A. E.: Marcott. C.: Story. G. M.: Ozaki. Y. Appl. Spectrose. 2000. 54.236A

13. Noda. I. Appl. Spectrose. 2000. 54. 994.

14. Thomas. E. V.: Haaland, D. M. Anal. Chem 1990, 62. l(1091.

15. Adachi. D.: Katsumoto, Y: Sato, H.: Ozaki. Y. Appl. Spectrosc. 2002. 56,357

16. Chun1g. H.: Lee. I. S.: Ku. M. S. Appl. Spectrosc. 1998. 52.885 